\title{
$N$-like rheograms of concentrated suspensions of magnetic particles
}

\author{
Modesto T. Lopez-Lopez, ${ }^{1 *}$ Laura Rodriguez-Arco, ${ }^{1}$ Andrey Zubarev, ${ }^{2}$ Pavel Kuzhir, ${ }^{3}$ \\ Larisa Iskakova $^{2}$, Fernando Gonzalez-Caballero ${ }^{1}$ \\ 1 Department of Applied Physics, Faculty of Science, Campus de Fuentenueva, \\ University of Granada, 18071 Granada, Spain \\ ${ }^{2}$ Urals Federal University, Lenina Ave 51, 620083, Ekaterinburg, Russia \\ ${ }^{3}$ Laboratory of Condensed Matter Physics, University of Nice-Sophia Antipolis,
} CNRS, UMR No. 7336, 28 Avenue Joseph Vallot, 06100 Nice, France

\begin{abstract}
We investigate the rheograms of concentrated suspensions of magnetic particles obtained under imposed shear rate in parallel plate geometry. We show that under magnetic field application the usual trend of the rheogram, i.e., increasing shear stress for the whole range of shear rates, is altered by the appearance of a region in which the shear stress decreases as the shear rate is increased. The existence of this region gives to the rheograms an $N$-like shape. The two initial regions (pre-yield regime) of these $N$ like rheograms present unstable flow, characterized by the oscillation of the shear stress with time for each imposed value of shear rate. We also show that rheograms obtained at different sample thicknesses approximately overlap in the developed flow regime, whereas there is a tendency of the shear stress to increase as the thickness is decreased in the pre-yield regime. This tendency is likely due to the strengthening of preexisting particle structures by compression as the gap thickness is decreased. Finally, we analyze the effect of the applied magnetic field strength, $H$, and demonstrate that the rheograms
\end{abstract}

\footnotetext{
*Author to whom correspondence should be addressed; electronic mail: modesto@ugr.es
} 
scale with $H^{1.5}$ to a single master curve, for the range of applied magnetic fields under study.

\section{INTRODUCTION}

Magnetorheological (MR) fluids are suspensions of micron-sized particles of magnetizable materials. These suspensions are characterized by the tunability of their rheological properties by means of magnetic field application, a phenomenon known as the MR effect [1]. Changes provoked in MR fluids by application of magnetic fields are so intense that their mechanical behavior usually shifts from this of a Newtonian liquid in their absence, to this of a plastic material in their presence [1]. Plastic materials are characterized by a minimum value of stress that must be applied in order to initiate their flow, known as the yield stress. In the case of MR fluids the magnitudes of both the yield stress and the viscosity increase when the intensity of the magnetic field is increased. This smart property is the base of many applications of MR fluids in engineering and biomedicine [2].

Rheology of MR fluids has been an active field of research since the eighties of the twentieth century. Main attention has been paid to the change of the characteristic rheological properties (yield stress, viscosity and viscoelastic moduli) with the intensity of the applied magnetic field; i.e., to the MR effect. The aim has usually been the experimental characterization of new MR fluids, and the understanding from the viewpoint of the particle interactions of the physical origins and strength of the observed MR effect. Recently, MR fluids constituted by fiber-like particles were prepared [3,4] and their rheology was extensively studied afterwards [5-8]. These studies revealed some unusual rheological behavior, which had not been shown before by any other kind of MR fluids. This is the case, for example, of a region of negative 
differential viscosity, characterized by a decrease of the shear stress as the shear rate is increased [8]. This kind of phenomena has stimulated the search of other unconventional rheological behaviors in MR fluids.

Very recently, we have found that near the limit of maximum-packing fraction, conventional (based on spherical particles) MR fluids also display a region of negative differential viscosity [9]. It is well documented that a stable flow is impossible within the shear rate range corresponding to the negative differential viscosity [10] and, in fact, we also found that for a given value of the shear rate pertaining to this region, the shear stress oscillated with time, in a way that resembled dry stick-slip motion [9]. In the present work, we focus on the analysis of the complete flow curves of concentrated MR fluids. As we show below, the region of negative differential viscosity gives to the rheograms an $N$-like shape, not observed before for other types of MR fluids constituted by spherical particles. We also analyze in the present work the effect of the measuring system gap thickness on the $N$-like rheograms. Interestingly, there is a tendency of the shear stress to increase as the gap thickness is decreased in the pre-yield regime. Finally, we analyze the effect of the strength of the applied magnetic field, $H$, and obtain an increase of the shear stress with $H^{1.5}$ for each given value of the shear rate, for the whole region of shear rate values.

\section{MATERIALS AND METHODS}

For the preparation of the MR fluids we used carbonyl iron powder (Fe-CC, BASF, Germany) as solid phase and mineral oil (Sigma Aldrich, Germany) as carrier liquid. According to the manufacturer, Fe-CC particles have a silica coating and are spherical in shape with a median diameter of $5 \mu \mathrm{m}$. We prepared MR fluids with 50 vol.\% approximate concentration, following the protocol described in [11]. For 
confirmation of some hypothesis that we will discuss in Sec. III, we also prepared MR fluids containing 10 vol.\% of Fe-CC particles, by dispersing proper amounts of the powder in mineral oil.

We characterized the rheological properties of our suspensions at $25^{\circ} \mathrm{C}$. In order to show that the main findings of our work are not the result of a particular experimental setup, we used two different rheometers and three different measuring geometries, as described in what follows.

(i) Haake Mars III provided with parallel plates with smooth surfaces. If not specified otherwise, data shown below were obtained with a Haake Mars III rheometer and measuring system geometry consisting of a set of parallel plates, $35 \mathrm{~mm}$ in diameter, with smooth surfaces. In particular, we used this setup for the study of the effect of gap thickness, which we varied between 0.1 and $1 \mathrm{~mm}$. For each gap thickness, we calibrated the rheometer by using two viscosity oil standards (Brookfield, USA) of $1.930 \mathrm{~Pa} \cdot \mathrm{s}$ and $6.470 \mathrm{~Pa} \cdot \mathrm{s}$, to ensure that the measurements obtained at the different gap thicknesses could be compared safely.

(ii) Haake Mars III provided with parallel plates with magnetic surface. In order to prove that the findings reported here do not qualitatively result as a consequence of the lack of transmission of shear forces to the measuring geometry due to the formation of a slip layer close to the smooth surface of the moving plate, we performed experiments with setup described in case (i) above, by previously coating the working surfaces by a thin magnetic layer note that this approach completely mitigates the problem of slip on the surface of the rheometer plates (wall slip) [12]. For this aim we glued a layer of Fe-CC particles to each of the working surfaces of the set of parallel 
plates. We checked at the end of the rheological measurements that the layers of Fe-CC particles were still glued to the working surfaces. We worked in this case with a gap thickness of $1 \mathrm{~mm}$.

(iii) Physica-Anton Paar MCR 300 provided with parallel plates with serrated surfaces. A general approach to avoid wall slip is the use of measuring systems with milled, serrated, or otherwise roughened or textured surfaces $[13,14]$. We also tested this approach and for this aim we used a rheometer Physica-Anton Paar MCR 300 provided with a measuring system consisting of a set of parallel plates, $20 \mathrm{~mm}$ in diameter, with serrated surfaces (see Figure 1). We worked in this case with a gap thickness of $0.35 \mathrm{~mm}$.

For the application of the magnetic field we used solenoids placed co-axially with the measuring geometry, as described in [15]. The experimental protocol used for the rheological measurements is reported in details elsewhere [9]. Briefly, we proceeded as it follows. First of all, we subjected the MR fluid to a linear shear rate ramp in the range $0.1-100 \mathrm{~s}^{-1}$, for a total duration of $60 \mathrm{~s}$. Then, we applied a magnetic field of a desired strength and we left the MR fluid at rest for 30 s. Finally, we imposed a shear rate (within the range $0.0025-100 \mathrm{~s}^{-1}$ ) and monitored the shear stress response for 30 minutes. When the measurement at a given shear rate was over, we replaced the sample by a fresh one and repeated the whole process for another shear rate.

\section{EXPERIMENTAL RESULTS}

\section{A. Time evolution of the shear stress}

Under magnetic field application, the time evolution of the shear stress of the MR fluid containing 50 vol. $\%$ of Fe-CC revealed that a steady value of the shear stress was not reached even at times as high as 30 minutes, for values of the imposed shear 
rate below a critical threshold (Figure 2a). As observed, after an initial sharp increase of the shear stress, some saw-tooth-like oscillations appeared at low enough values of the imposed shear rate. In a previous work we studied the origin of these oscillations and showed that from a microscopic viewpoint they were the consequence of the periodic failure and healing of the field-induced particle structures [9]. In more details, we supposed the appearance of a periodic array of gap-spanning cylindrical columns. These columns were tilted by the motion of the upper rheometer plate, and broken at their middle point at some critical tilt angle (corresponding to the maximum of the stress vs. strain curve). Afterwards, the two halves of the broken aggregates were supposed to be reformed with other broken columns at the first encounter. The temporal period of the stress oscillations was therefore directly related to the space period of the column-like structure. For more details on the microscopic origin of the stress oscillations see [9].

As discussed in the section materials and methods we checked that the stress oscillations were not the result of a particular instrumental setup. As observed (see inset of Figure 2a), stress oscillations were also obtained for measuring plates with a magnetic layer coating. In addition, we previously reported similar stress oscillations for measurements at imposed shear rate using rheometer Physica-Anton Paar MCR 300 provided with parallel plates with serrated surfaces [9], which confirms the independence on the experimental setup of our results from the qualitative point of view. Furthermore, note that similar stress oscillations under simple shear for MR fluids consisting of $30 \mathrm{vol} . \%$ of iron in silicone oil were very recently reported by other authors [16]. Surface roughness of the measuring plates was approx. 0.02 microns, whereas iron particles were 3 microns in diameter in this previous work [16].

Contrarily to concentrated suspensions, MR fluids containing 10 vol.\% of Fe-CC particles did not show stress oscillations, but a steady state regime with an 
approximately constant value of the shear stress achieved at large enough times (Figure 2b). This fact confirms our previous hypothesis [9] that stress oscillations observed for highly concentrated MR fluids were a consequence of the stick-slip motion, provoked by the periodic fracture and reformation of the thick 3-dimensional structure induced by the applied field. Note that, for some reason, collective breaking and reformation of aggregates did not occur in suspensions of moderately low particle volume fraction (e.g., 10 vol.\%). This was likely because of irregular spacing between columns, which would hinder the collective behavior more easily at low concentrations (when the distance between aggregates was large) than at high concentrations.

Note at this point, that during the decreasing part of the stress oscillations, the imposed value of the shear rate was not maintained (Figure 3). As observed, during the increasing part, the shear rate was perfectly constant and the shear stress monotonically increased. This increase of the shear stress corresponded with the deformation of the 3 dimensional particle structure induced by the field. Deformation of this structure required a work, made by the shearing force. At the maximum of the stress within each oscillation, the 3-dimensional particle structure fractured -experimental evidence, as well as detailed discussion, of the microscopic origin of the stress oscillations can be found in our previous work [9]. Slip of one part of the fractured structure over the other part required much lower shear stress than deformation of the field-induced particle structure and, consequently, the shear rate increased sharply during a short time interval due to the inertia of the upper plate of the rheometer. We refer here to slip occurring between broken aggregates, not to slip between aggregates and the rheometer wall (wall slip). Note that the rheometer was unable to react immediately to the new situation (slip between broken structures) that required much lower shear stress and, consequently, for a short time interval the stress applied provoked a sharp increase of the rotation speed of 
the upper plate (and thus of the shear rate), as observed in Figure 3. The augmented values of the shear rate elapsed the total duration of the decreasing part of the stress oscillation, presumably until the particle structures reformed again, which marked the onset of the next increasing shear stress region. Both the peak value of the shear rate (with respect to the imposed value), and the duration of the decreasing part of the stress oscillation, decreased with the increase of the imposed value of the shear rate (Figure 3 ). For the values of applied magnetic field used in our work, the shear rate, $\dot{\gamma}$, was maintained within a maximum deviation of approx. $10-20 \%$ of its imposed value for $\dot{\gamma} \geq 0.5 \mathrm{~s}^{-1}$ for rheometer Haake Mars III and for $\dot{\gamma} \geq 0.025 \mathrm{~s}^{-1}$ for rheometer PhysicaAnton Paar MCR300 - obviously, this error was much smaller the higher the value of the imposed shear rate, being negligible close and above the yield point.

Such sharp increase of the shear rate was not observed for moderately diluted MR fluids. For these suspensions the shear rate was approximately maintained around the imposed value, as exemplified in Figure 4.

\section{B. $N$-like shaped rheograms}

The analysis of the segments of the stress vs. time curves (like those shown in Figure 2) at large elapsed times and for each value of the imposed shear rate allowed us to obtain the steady-state rheograms (curves of shear stress vs. shear rate) of our suspensions. For those experimental conditions that gave rise to oscillating values of the shear stress, we took the mean value of the stress (within its oscillations) as the representative stress for the corresponding shear rate -note at this point that if restricted to the shear stress values corresponding to the increasing part of the oscillations, for which the shear rate was perfectly maintained, the same rheograms were obtained. 
Under an applied magnetic field, the rheograms of suspensions containing $50 \mathrm{vol} . \%$ of iron particles presented a marked $N$-like shape, characterized by an initial increase of the shear stress with the shear rate, followed by a decrease of the shear stress as the shear rate was further increased, and a final, rather strong, increase of the shear stress for values of the shear rate higher than approx. $10 \mathrm{~s}^{-1}$ (Figure 5b). Similar $N$-like rheograms were also obtained for measuring plates with rough surfaces, as well as for measuring plates with a magnetic layer coating (Figure 5c). However, as observed, values of the shear stress for a given value of the shear rate were considerably higher for measuring plates with a magnetic layer coating. This enhancement of the MR effect can be explained as a result of the better transmission of forces to the working plate, in agreement with previous works $[12,17]$. On the other hand, the $N$-like shape was not so significant in the absence of applied magnetic field (Figure 5a). Furthermore, it was not appreciably detected in rheograms of suspensions containing 10 vol. $\%$ of iron under an applied magnetic field (Figure 5d).

Note that similar $N$-like rheograms to these of suspensions of 50 vol.\% of iron particles under applied magnetic field were reported for laponite suspensions [18]. Very recently, we also observed similar rheograms for suspensions of magnetic nanofibers [8], although in this previous work the decreasing region of shear stress was obtained at much higher values of the shear rate (around $10 \mathrm{~s}^{-1}$ ). The decrease of the shear stress with the shear rate in the case of magnetic nanofibers was connected to the existence of strong friction between particles.

\section{Effect of the gap thickness of the measuring system}


Concerning the effect of measuring system gap on the rheograms of concentrated MR fluids (50 vol.\%), in the absence of applied magnetic field rheograms obtained at different gap thicknesses approximately superimposed for the whole range of shear rates (Figure 5a). On the contrary, when a magnetic field was applied, there were differences in the rheograms obtained at different gap thicknesses (Figure 5b). As observed, in the pre-yield regime (initial increasing part of the shear stress and decreasing part of the shear stress) there was a tendency of the shear stress to increase as the gap thickness was decreased. On the other hand, in the flow regime (shear rate higher than $1 \mathrm{~s}^{-1}$ ) all the curves approximately superimposed.

The enhancement of magnetic field-induced yield stress as the gap thickness was decreased was previously observed for concentrated MR suspensions subjected to compression $[19,20]$. In those cases, compression of pre-existing structures gave rise to thicker particle structures, which resulted in stronger MR effect.

Something similar could be the cause for the enhancement of the shear stress in the pre-yield regime as the gap thickness was decreased, observed in our experiments note that in the flow regime particle structures are broken. To check the validity of this hypothesis, we submerged the rotating plate of the Haake Mars III rheometer in a bath (cup) containing the concentrated MR fluid (50 vol.\% of iron particles), applied a magnetic field of $5.4 \mathrm{kA} / \mathrm{m}$, and fixed a shear rate of $0.05 \mathrm{~s}^{-1}$. Under the applied magnetic field and continuous rotation of the plate, we decreased the gap, from $1 \mathrm{~mm}$ down to $0.4 \mathrm{~mm}$, stepwise, and monitored the evolution of the shear stress and the normal force (Figure 6).

As observed, the normal force increased abruptly when the gap thickness was decreased, although it relaxed afterwards (Figure 6). The shear stress also increased 
abruptly when the gap thickness was decreased and then experienced some oscillations in coherence with observations of Figure 2. Note that relaxation of the normal force was very sharp (stepwise) at some specific points that corresponded to the abrupt decrease of the shear stress and, thus, to the breakage of the particle structures into two parts. This result corroborates the hypothesis that under an applied magnetic field, compression results in stronger MR structures. Nevertheless, in the experiments of Figures 2-5 we compressed our samples without shear and without magnetic field application.

To check if the same phenomenon of structure enhancement could take place under these conditions, we measured the normal force on the upper, rotating plate, submerged in the concentrated MR fluid, contained in the cup, this time without magnetic field and without shear application. Results show that the normal force increased abruptly when the gap thickness was decreased, and relaxed smoothly with time afterwards (Figure 7). The behavior observed in Figure 7 can be explained as it follows. In our concentrated suspensions, very likely there were preexisting particle aggregates as a consequence of colloidal interactions and remnant magnetization. When the gap thickness was decreased, these aggregates would have been compressed, which would explain the observed increase of the normal force (Figure 7). Such compressive pre-load at zero field would have resulted in the interpenetration of asperities of touching particles. Once the field was applied, these particles would have experienced a stronger magnetic attraction than those for which compression was not performed. The lower the gap, the stronger the compression of initial aggregates as inferred from Figure 7 and, therefore, the stronger would have been the interpenetration of the particle asperities, giving rise to a stronger shear stress under magnetic field in the pre-yield regime, in agreement with results of Figure $5 b$.

\section{Effect of applied magnetic field strength}


We also analyzed the effect of the applied magnetic field on the rheograms and, as observed (see Figure 8a), we obtained similar $N$-like shaped rheograms for different applied field strengths, with a tendency of the shear stress to increase as the magnetic field strength was increased, as expected for a MR fluid. It is worthy to mention at this point that in a previous paper [11] we developed a model for the magnetic field-induced yield stress of highly concentrated MR fluids.

It is well-known that the yield stress of MR fluids scales as $\mu_{0} M_{s}^{0.5} H^{1.5}$, with the magnetic field intensity $H$, for applied fields of medium intensity (far from the initial linear region of the magnetization curve of the material and from the saturation region) [1]. Here $\mu_{0}$ is the vacuum permeability and $M_{s}$ the saturation magnetization of the magnetic particles of the MR fluid. We used this scaling and, as observed, the curves for the different applied field strengths approximately superimpose in a single master curve (Figure 8b). For this purpose, we used the saturation magnetization of the Fe-CC particles, $M_{s}=870.9 \pm 1.4 \mathrm{kA} \mathrm{m}^{-1}[14]$. We can thus conclude that in concentrated MR fluids, for each imposed value of the shear rate, the resulting shear stress approximately scales with $H^{1.5}$.

\section{CONCLUSIONS}

We have shown that in the pre-yield regime a steady-state flow is impossible in highly concentrated suspensions of magnetic particles subjected to an applied magnetic field. At a given value of the imposed shear rate within the pre-yield regime, instead of a quasi-constant value of the shear stress at large enough times, we obtained shear stress oscillations of well-defined amplitude and period. Rheograms obtained from the experimental measurements displayed an $N$-like shape, with the initial (at very low 
shear rate) increasing region of stress and the decreasing one, corresponding to the values of shear rates for which oscillations were observed. The final increasing region of stress, at high enough values of the shear rate, corresponded to the regime of developed flow, for which no oscillations were obtained. Both stress oscillations and the $N$-like shape disappeared in the absence of applied field and were not observed under any experimental condition for suspensions of moderately low particle concentration.

Concerning the effect of the measuring system gap thickness on the obtained $\mathrm{N}$ like rheograms, there was overlap of the rheograms in the developed flow regime. However, in the pre-yield regime, the shear stress tended to increase as the gap thickness was decreased. This increment was very likely related to an enhancement of particle structures under compression, as supported by the increase of normal force that we obtained experimentally. Finally, we have also shown that for a given value of the imposed shear rate the resulting shear stress increased as $H^{1.5}$, with the magnetic field intensity $H$, for the range of magnetic fields used in our work.

\section{ACKNOWLEDGEMENTS}

This work was supported by project FIS2013-41821-R, MINECO, Spain; by project 3.12.2014/K, Program of Ministry of Science and Education of the Russian Federation; by the Act 211 Government of the Russian Federation, contract № 02.A03.21.0006; by grants of RFFI 13-02-91052, 13-01-96047 (Ural) and 14-08-00283; and by project PICS 6102 CNRS/Ural Federal University. L.R.-A acknowledges financial support from University of Granada.

\section{References}


[1] Bossis, G., O. Volkova, S. Lacis, and A. Meunier, "Ferrofluids," Lect. Notes Phys. 594, 201-230 (2002).

[2] Park, B. J., F. F. Fang, and H. J. Choi, "Magnetorheology: materials and application," Soft Matter 6, 5246-5253 (2010).

[3] Bell, R. C., J. O. Karli, A. N. Vavreck, D. T. Zimmerman, G. T. Ngatu, and N. M. Wereley, "Magnetorheology of submicron diameter iron microwires dispersed in silicon oil," Smart Mater. Struct. 17, 015028 (2008).

[4] López-López, M. T., G. Vertelov, P. Kuzhir, G. Bossis, and J. D. G. Durán, "New magnetorheological fluids based on magnetic fibers," J. Mater. Chem. 17, 38393844 (2007).

[5] López-López, M. T., P. Kuzhir, and G. Bossis, "Magnetorheology of fiber suspensions. I. Experimental,” J. Rheol. 53, 115-126 (2009).

[6] de Vicente, J., J. P. Segovia-Guitérrez, E. Anablo-Reyes, F. Vereda, and R. HidalgoAlvarez, "Dynamic rheology of sphere- and rod-based magnetorheological fluids," J. Chem. Phys. 131, 194902 (2009).

[7] Bell, R. C., D. Zimmerman, and N. M. Wereley, "Impact of nanowires on the properties of magnetorheological fluids and elastomer composites," in Electrodeposited Nanowires and Their Applications, edited by N. Lupu (Intech, Vienna, Austria, 2010), Chap. 8, pp. 189-212.

[8] Zubarev, A., M. T. López-López, L. Iskakova, and F. González-Caballero, "N-Like rheograms of suspensions of magnetic nanofibers," Soft Matter 9, 1902-1907 (2013).

[9] López-López, M. T., P. Kuzhir, L. Rodríguez-Arco, J. Caballero-Hernández, J.D.G. Durán, and G. Bossis, "Stick-slip instabilities in the shear flow of magnetorheological suspensions," J. Rheol. 57, 1101-1119 (2013). 
[10] Quemada, D., “Unstable flows of concentrated suspensions," Lect. Notes Phys. 164, 210-247 (1982).

[11] López-López, M. T., P. Kuzhir, J. Caballero-Hernández, L. Rodríguez-Arco, J.D.G. Durán, and G. Bossis, "Yield stress in magnetorheological suspensions near the limit of maximum-packing fraction," J. Rheol. 56, 1209-1224 (2012).

[12] Laun, H. M., C. Gabriel, and C. Kieburg, "Wall material and roughness effects on transmittable shear stresses of magnetorheological fluids in plate-plate magnetorheometry," Rheol. Acta 50, 141-157 (2011).

[13] Buscall, R., J. I. McGowan, and A. J. Morton-Jones, “The rheology of concentrated dispersions of weakly attracting colloidal particles with and without wall slip," J. Rheol. 37, 621-642 (1993).

[14] Gómez-Ramírez, A., M. T. López-López, F. González-Caballero, J. D. G. Durán, "Wall slip phenomena in concentrated ionic liquid-based magnetorheological fluids," Rheol. Acta 51, 793-803 (2012).

[15] Kuzhir, P., M. T. López-López, G. Vertelov, C. Pradille, and G. Bossis, "Shear and squeeze rheometry of suspensions of magnetic polymerized chains," Rheol. Acta 47, 179-187 (2008).

[16] Jiang, J., G. Hu, Z. Zhang, Y. Meng, Y. Tian, "Stick-slip behavior of magnetorheological fluids in simple linear shearing mode," Rheol. Acta 54, 859-867 (2015).

[17] Jonkkari, I., E. Kostamo, J. Kostamo, S. Syrjala and M. Pietola, "Effect of the plate surface characteristics and gap height on yield stresses of a magnetorheological fluid," Smart Mater. Struct. 21, 075030 (2012). 
[18] Pignon, F., A. Magnin, and J. M. Piau, "Thixotropic colloidal suspensions and flow curves with minimum: identification of flow regimes and rheometric consequences,” J. Rheol. 40, 573-587 (1996).

[19] Tang, X., X. Zhang, R. Tao, and Y. Rong, "Structure-enchanced yield stress of magnetorheological fluids,” J. Appl. Phys. 87, 2634-2638 (2000).

[20] Tao, R., "Super-strong magnetorheological fluids," J. Phys.: Condens. Matter, 13, R979-R999 (2001). 


\section{Figure Captions}

Figure 1. Sketch of the rough (serrated) geometry used with MCR 300 (Physica-Anton Paar) rheometer. Reprinted with permission from [14].

Figure 2. Time evolution of the shear stress for different imposed values of the shear rate (indicated in the figure). Gap thickness between measuring plates was $1 \mathrm{~mm}$; applied magnetic field strength was $12 \mathrm{kA} / \mathrm{m}$. (a) MR fluid containing 50 vol.\% of Fe$\mathrm{CC}$-inset shows results for measurement at an imposed shear rate of $0.05 \mathrm{~s}^{-1}$ with rheometer Haake Mars III provided with magnetic-coated plates; (b) MR fluid containing 10 vol.\% of Fe-CC.

Figure 3. Shear rate and shear stress plotted as a function of shearing time for MR fluids containing $50 \mathrm{vol} \%$ of Fe-CC particles under application of a magnetic field of $12 \mathrm{kA} / \mathrm{m}$. Gap thickness: $1 \mathrm{~mm}$; imposed (mean) value of the shear rate: (a) and (b) $0.0025 \mathrm{~s}^{-1}$; (c) and (d) $0.5 \mathrm{~s}^{-1}$. (b) and (d) focus on brief time intervals.

Figure 4. Shear rate and shear stress plotted as a function of shearing time for a MR fluid containing 10 vol.\% of Fe-CC particles under application of a magnetic field of 12 $\mathrm{kA} / \mathrm{m}$. Gap thickness: $1 \mathrm{~mm}$; prefixed value of the shear rate: $0.005 \mathrm{~s}^{-1}$.

Figure 5. Rheograms of MR suspensions. (a) In the absence of applied magnetic field, $H$, for a suspension containing $50 \mathrm{vol} . \%$ of iron particles. (b) Under $H=12 \mathrm{kA} / \mathrm{m}$ for a suspension containing 50 vol.\% of iron particles. Gap thickness in (a) and (b): $\mathbf{a}: 0.1$ $\mathrm{mm} ; \boldsymbol{\Delta}: 0.4 \mathrm{~mm} ; \boldsymbol{\nabla}: 1 \mathrm{~mm}$. (c) Under $H=12 \mathrm{kA} / \mathrm{m}$ for a suspension containing 50 vol.\% of iron particles using rheometer Haake Mars III provided with magnetic-coated plates ( $\bullet$ ) and rheometer Physica Anton Paar provided with serrated plates ( $\boldsymbol{\square})$. (d) Under $H=12 \mathrm{kA} / \mathrm{m}$ for a suspension containing $10 \mathrm{vol} . \%$ of iron particles. 
Figure 6. Time evolution of the normal force (a) and the shear stress (b), as the gap thickness between the rotating plate and the bottom of the stationary cup was decreased stepwise, as quantified in the figure. During the whole experiment the rotating plate was submerged in a concentrated MR fluid (50 vol.\% of iron particles), subjected to an applied magnetic field of $5.4 \mathrm{kA} / \mathrm{m}$ of strength.

Figure 7. Time evolution of the normal force as the gap thickness between the rotating plate and the bottom of the stationary cup was decreased stepwise, as quantified in the figure. During the whole experiment the rotating plate was submerged in a concentrated MR fluid (50 vol.\% of iron particles). There was no magnetic field application.

Figure 8. Rheograms of a suspension containing 50 vol. $\%$ of iron particles under the effect of different values of the strength of the applied magnetic field, $H$. (a) Shear stress, $\sigma$, as a function of shear rate, $\mathrm{d} \gamma / \mathrm{dt}$. (b) Normalized shear stress, $\sigma \mu_{0}^{-1} M_{s}^{-0.5} H^{-1.5}$, as a function of shear rate, $\mathrm{d} \gamma / \mathrm{dt}$-in this formula, $\mu_{0}$ is the vacuum permeability and $M_{s}$ the saturation magnetization of the iron powder. Measurements were carried out with rheometer Physica Anton Paar provided with serrated surfaces. The symbols correspond to the experimental data; the continuous lines are eye guidelines. Applied magnetic field strength: $\boldsymbol{\square}: 0 \mathrm{kA} / \mathrm{m} ; \bullet: 9 \mathrm{kA} / \mathrm{m} ; \boldsymbol{\Delta}: 12 \mathrm{kA} / \mathrm{m} ; \boldsymbol{\nabla}$ : $19 \mathrm{kA} / \mathrm{m} ;: 27 \mathrm{kA} / \mathrm{m}$. 

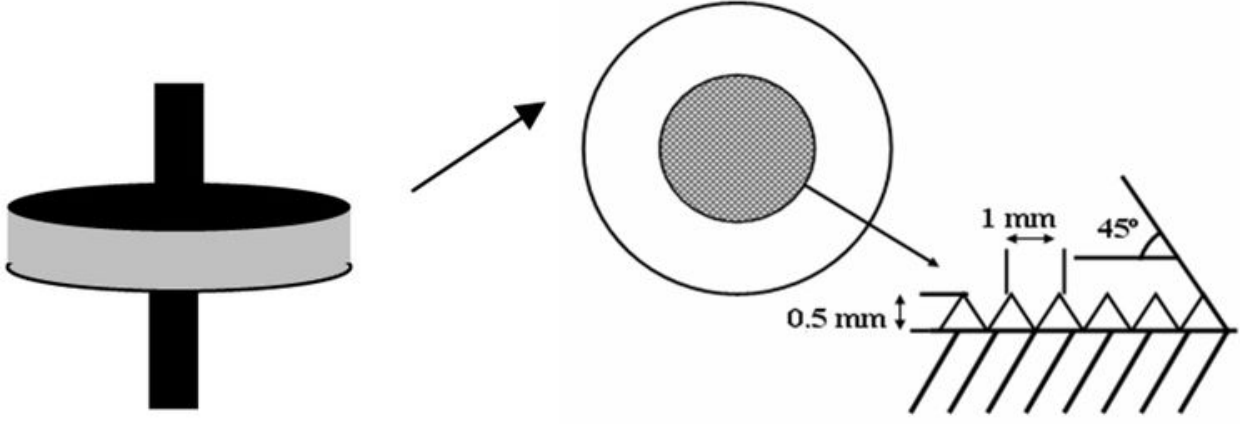

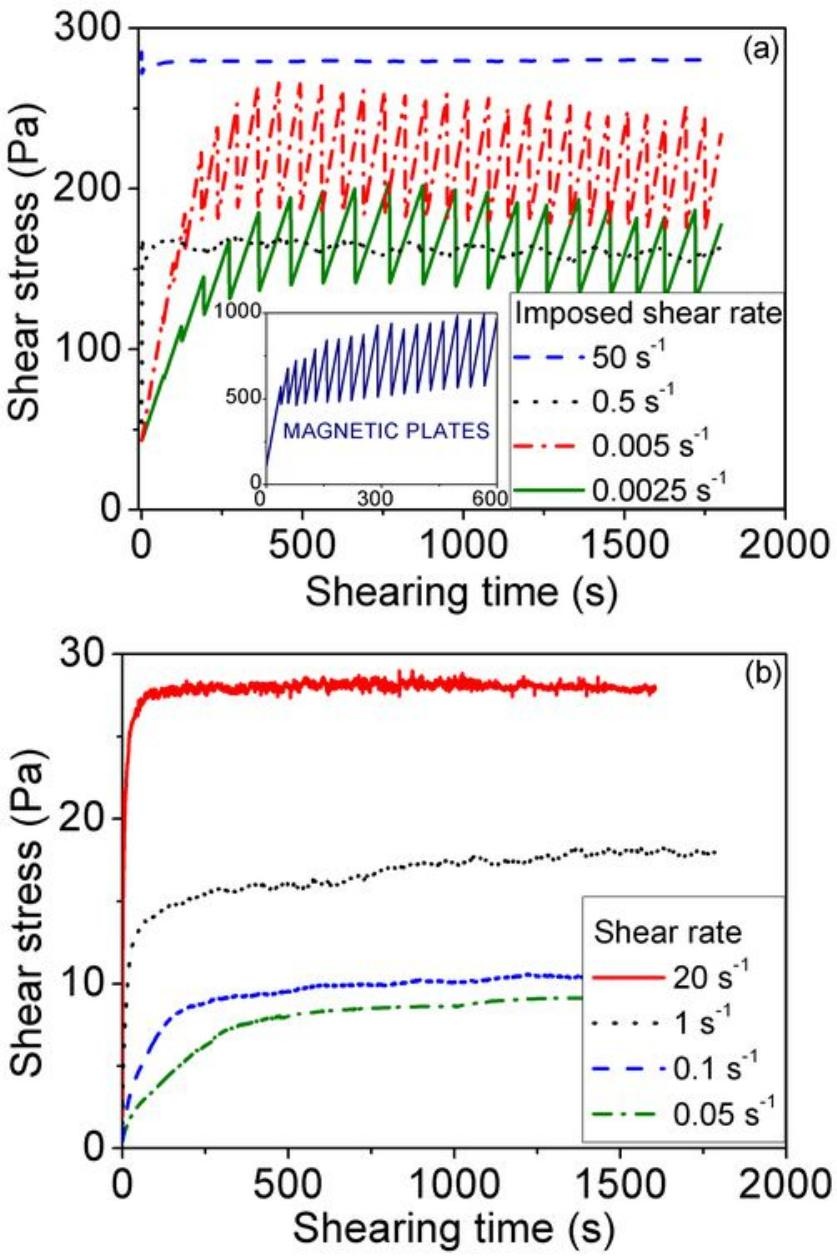


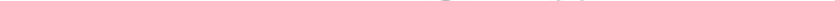



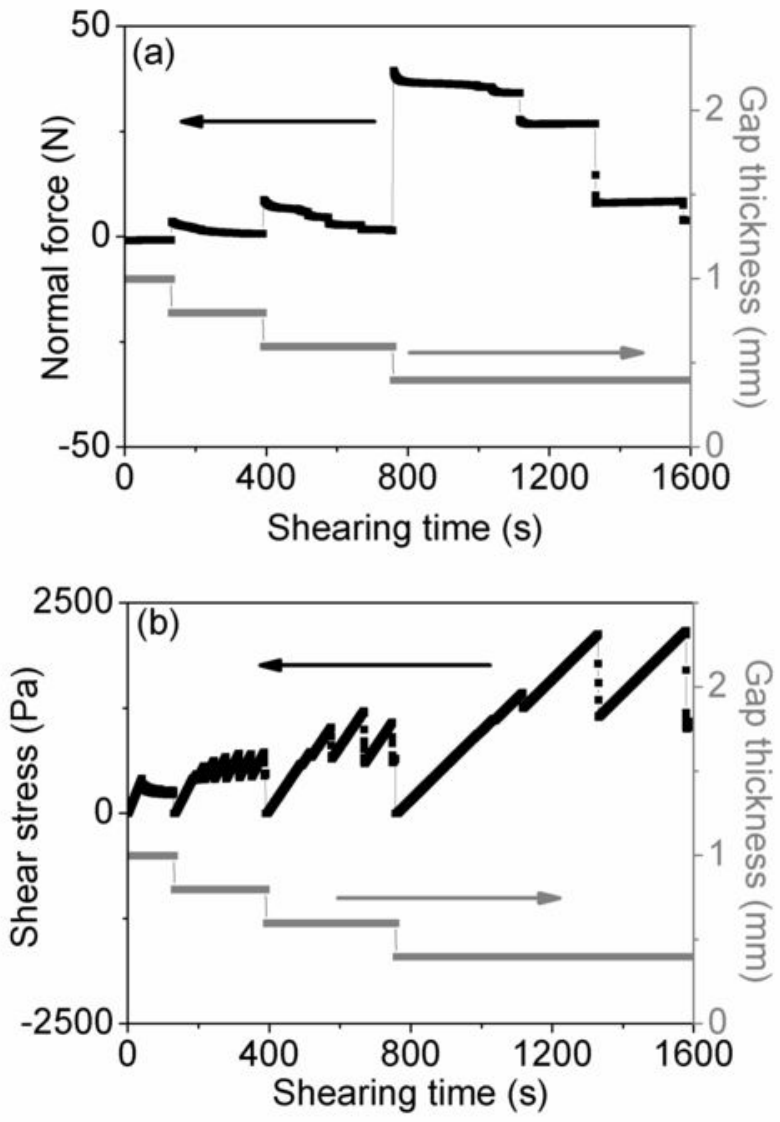


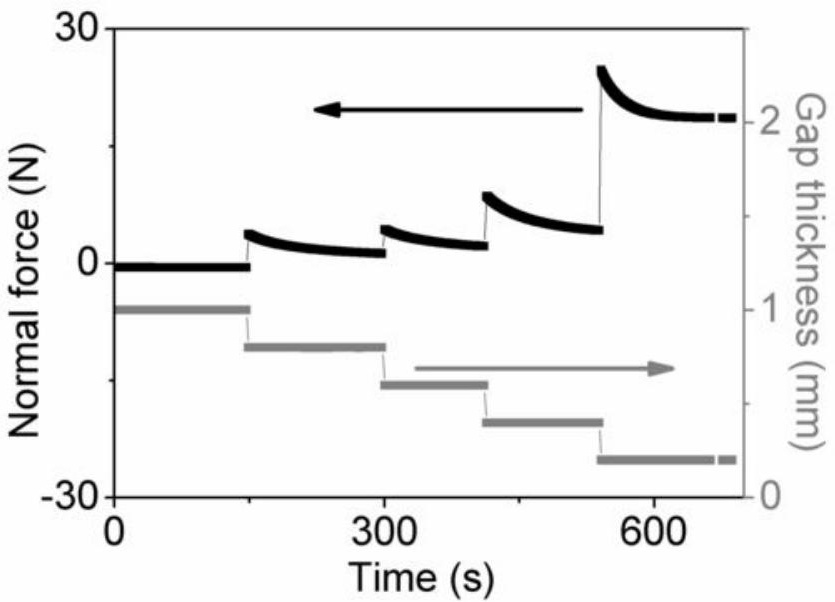



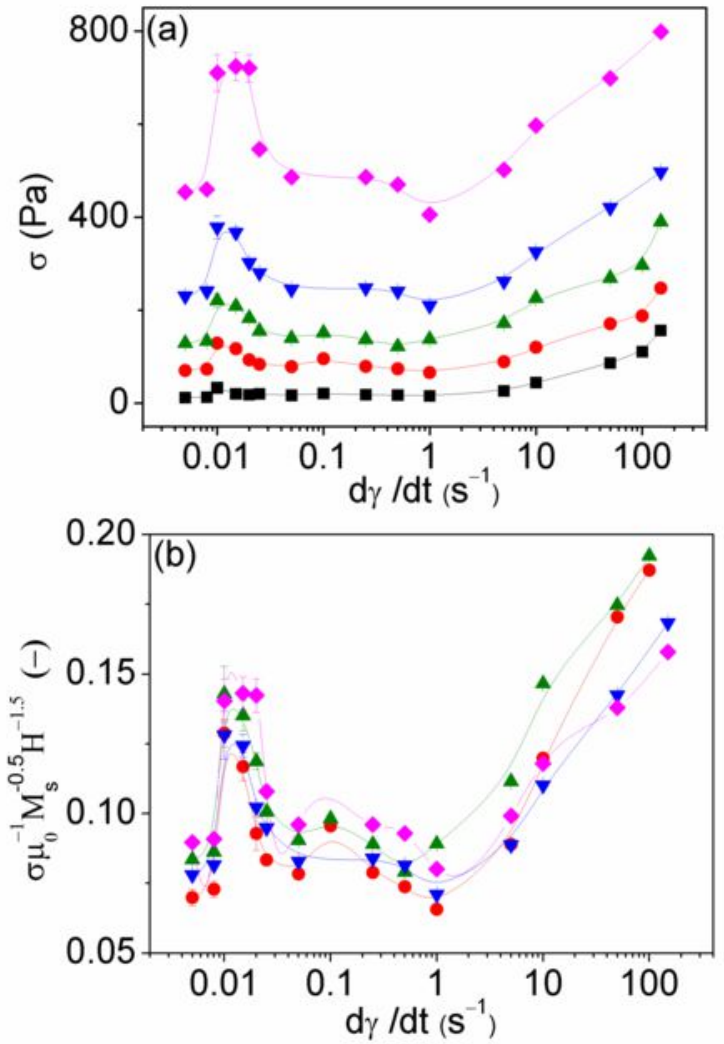